Voix et Images

voixetimages

\title{
Jacques Ferron et les écrivains
}

\section{Donald Smith}

Volume 8, numéro 3, printemps 1983

Jacques Ferron

URI : https://id.erudit.org/iderudit/200400ar

DOI : https://doi.org/10.7202/200400ar

Aller au sommaire du numéro

\section{Éditeur(s)}

Université du Québec à Montréal

\section{ISSN}

0318-9201 (imprimé)

1705-933X (numérique)

Découvrir la revue

\section{Citer cet article}

Smith, D. (1983). Jacques Ferron et les écrivains. Voix et Images, 8(3), 437-453.

https://doi.org/10.7202/200400ar d'utilisation que vous pouvez consulter en ligne.

https://apropos.erudit.org/fr/usagers/politique-dutilisation/ 


\title{
Jacques Ferron et les écrivains:
}

\author{
par Donald Smith, Universite Carleton
}

Ferron sera peut-être reconnu un jour comme un des plus grands conteurs contemporains de langue française. S'il est vrai que Ferron n'a publié que deux recueils de contes proprement dits, tous ses romans, a l'exception du Ciel de Québec, son seul véritable roman, sont écrits sur le mode du conte. La perception ferronienne des êtres et des choses, fondée sur la fantaisie, le féerique, le merveilleux et l'ironie, relève du style ramassé et chargé des conteurs. Lorsque Ferron traite de l'histoire, par exemple, c'est l'historiette qui prend le dessus. Or, I'historiette s'appacente au conte amusant et comique. Si bien que les Historiettes et les références historiques ne sont en fait qu'une extension de la vision d'un conteur militant et spirituel s'évertuant à exprimer ses idées sur la société. Jean Marcel le dit bien:

II y a, en effet, entre le conte et l'historiette des frontières qui sont mal tracées. Chez Ferron, l'historiette est un "conte à charge", comme on dit "un portrait à charge"; elle est pour tout dire, un conte, mais polémique, qui n'utilise l'histoire que comme appoint d'une liberté plus haute. L'exiguñté de son champ esthé tique va favoriser l'association, la souplesse, la modulation passant du fait historique à la fantaisie $(. .)^{1}$.

Le traitement de l'histoire chez Ferron a été profondément influencé par Antoine Hamilton. Dans les Mémoires du comte de Gramont ${ }^{2}$, le mémorialiste irlandais francisé sé met à la place de son ami Gramont pour écrire des chroniques audacieuses, cyniques et amusantes sur la polissonnerie, le vice, la corruption et les ridicules intrigues de l'amour dans le monde de la politique. Les acteurs du drame, personnages vrais de l'époque de la Restauration anglaise refondus par la fantaisie, s'animent pour illustrer l'hypocrisie du puritanisme et des intrigues politiques. Hamilton trouve toujours la'bonne formule, l'image, le raccourci pour faire passer sa satire. Chaque fois que Ferron met la main sur des personnages réls, hommes politiques, personnages historiques, écrivains, il reste fidèle à la fable "armée" d'Antoine Hamilton.

C'est dans une telle veine fabuleuse et transformationnelle qu'une cinquantaine d'écrivains sont incorporés dans l'oeuvre de Ferron. A travers eux, celui-ci approfondit ses idées sur la société et sur le pays. Parfois, il s'agit d'une mention passagère que le lecteur ne peut s'expliquer que par rapport à des auteurs discutés plus en détail. Mais nous trouvons également des pages entières consacrées à un roman ou à une 
pièce de théâtre. Dans chaque cas, les écrivains prennent soit une forme caricaturale (auteurs-antagonistes: Anne Hébert, introvertie strip-teaseuse; Saint-Denys Garneau, intimiste boutonneux; Jean Le Moyne, employé du "C.N. Raillerouillé limitée", etc.) soit exemplaire lauteurs protagonistes: Nelligan, héros irlandais enquébécoisé et antimatérialiste; Louis Hémon, dénonciateur de barbares impérialistes; Marie-Victorin, "ignorantin" éclairé et humaniste' amoureux d'une petite fougère, etc.).

Dans cette étude, il sera moins question de la littérature en général que de la façon dont elle devient pour Ferron une occasion de faire passer des idées sociales. De fait, les auteurs qu'il évoque tendent à perdre leur identité propre pour devenir des personnages de l'oeuvre littéraire ${ }^{3}$. Si l'on est parfois tenté de dire que Ferron est injuste, voire méchant à l'égard de tel ou tel écrivain, il ne faut pas oublier que l'écrivain en question est "récupéré", assimilé à l'univers ferronien. Un peu à la façon de Gérard Bessette, dans le Semestre, Ferron nous fait rire avec tous ses écrivains "thę tralisés", transformés, tout en nous faisant réfléchir aux implications idéologiques de leurs oeuvres par rapport à ses propres idées sur la société at sur le pays.

\section{Les antagonistes québécois}

Alfred Garneau, et surtout Saint-Denys Garneau, sont les deux écrivains qué bécois qui se font le plus malmener par Ferron. Ils sont présentés comme les apôtres d'une littérature désincarnée et anti-patriotique, trahissant l'idéal de leur homonyme, François-Xavier Garneau. La poésie d'Alfred Garneau, traducteur au Sénat et fils aîné de François-Xavier Garneau, est vue comme une poésie "intimiste" qui rapetisse à sour hait le paysage québécois:

Avec lui les mots d'importation, purement livresques, qui ne correspondent pas à notre réalité, font leur entrée au pays. "Un rossignol vint boire au flot harmonieux." Un rossignol, vous avez déjà vu ça, curé Rondeau? Ve Ciel de Québec, p. 182)

Accusant ironiquement Alfred Garneau de "ne jamais avoir pensé et d'avoir été "vieille fille" (le Ciel de Québec), Ferron proteste contre le fait que trop de poètes canadiens-français du dix-neivième siècle aient eu tendance à imiter servilement Musset, Lamartine et Hugo, qu'ils aient chanté une flore et une faune exotiques.

L'intimisme exotique est représenté également par Saint-Denys Garneau. Dès "Mélie et le boeuf" (1962). Saint-Denys Garneau est associé à un taureau malheureux pris dans une cage d'os, métaphore empruntée à un poème de Regards et Jeux dans l'espace:

Je suis pris dans une cage d'os. L'oiseau dans sa cage d'os, c'est la mort qui fait son nid. Naguère, j'esperais me libérer en écrivant, mais les poèmes que je fis alors ne rendaient pas mon cri. ("Mélie et le boeut", Contes du pays incertain, p. 37)

Cette référence a Saint-Denys Garneau ne peut s'expliquer que lorsqu'on la replace dans le contexte des idées historiques de Ferron. Le nom de famille de Saint-Denys Garneau évoque, par renvoi, François-Xavier Garneau qui devient, chez Ferron, le signe par excellence du Patriote, de l'engagement et de l'affirmation de soi. Saint-Denys 
Garneau, au contraire, écrit de la poésie métaphysique où les questions philosophiques et les thèmes de l'isolement et de l'absence priment sur l'affirmation de l'identité nationale.

Dans le Saint-Elias, sans identifier directement l'auteur du poème "Cage d'oiseau", le romancier revient a la charge, cette fois pour condamner l'indifférence et l'isolement des riches. On sait, en effet, que Saint-Denys Garneau est issu d'une famille aux lointaines origines aristocratiques. Marguerite Cossette, du Saint-Elias, s'attriste dans un manoir de Sainte-Catherine (évidernment celui des Juchereau-Duchesnay). Arriviste, victime de sa fortune, elle suffoque, "tel un oiseau sagace et muet dans une cage" (p. 181).

Saint-Denys Garneau apparaît épisodiquement dans plusieurs oeuvres de Ferron. II se mue en "fils" boutonneux de la "fofolle" Garneau (/e Saint-Elias) et en "grand boutonneux en lettres tourné vers le prestige" (la Chaise du maréchal ferrant). Dans le Coeur d'une mère, il prend la forme de Septime, sculpteur-poète, écrivain replié sur lui-même, à la recherche d'un idéal mystique, souffrant, dans la maison familiale, du "préjugé aristocratique".

C'est dans le Ciel de Québec que Ferron joue le plus avec ce persoinnage. Le romancier lui consacre de nombreuses pages où le poète, rebaptisé Orphée, est placé dans "l'enfer des indécis". Avec quelques amis mélomanes, peintres et théologiens, Orphée crée une "coterie" et une revue, la Re/ève, dont Ferron dit ironiquement que le nom "indique assez bien le caractère conservateur" (p. 175). L'équipe de la Relève, formée de "privilégiés d'une société en perdition, gens de loisir et non de chómage" (p. 175), se préoccupe de problèmes qui les rendent "fous de religion". Selon Ferron, ce sont des penseurs qui, faute de conscience sociale, " $n$ 'agissent pas". Ils tiendraient leur philosophie néothomiste de Jacques Maritain tout en idolătrant le mysticisme de Claudel", le spiritualisme de Péguy", et le catholicisme absolu de Léon Bloy". Ils "vont en retraite à la Trappe d'Oka pour communiquer directement avec le Boss sans passer par les Foremen", alors que les chômeurs "font des pèlerinages à l'Oratoire Saint-Joseph pour que le frère André leur trouve du travail" Ve Ciel de Québec, p. 175).

Si Robert Charbonneau fait une apparition rapide dans le Ciel de Québec, c'est surtout parce que l'auteur de Chronique de l'áge amer est "fort peu renseigne" sur la "trahison" de Sairt-Denys Garneau. Dans son roman autobiographique, Charbonneau raconte I'histoire des membres de la Reléve qui se réunissaient à Westmount pour discuter de musique et du "principe ridicule" du nationalisme souverainiste. L'auteur de la "relation de l'áge amer" (p. 185) prend beaucoup de plaisir à décrire le poète de Sainte-Catherine assis confortablement dans la maison de ses parents à Westmount et plus tard dans le manoir, sortant parfois, tel un oiseau blessé, pour "se voleter" sur la "charmante ile d'Orléans". Ferron affirme que si Charbonneau avait été mieux renseigné "du côté de Québec", il aurait compris que Saint-Denys Garneau faisait honte à l'ancètre François-Xavier, à l'écrivain modèle d'un Québec à la dérive.

Ferron exagère-t-il au sujet du présumé manque d'engagement social de l'auteur de "Cage d'oiseau"? II me semble que non. Saint-Denys Garneau a écrit plusieurs articles sur les romans de Chateaubriant, oeuvres lyriques et chrétiennes inspirées du terroir vendéen. Or, le mysticisme de Chateaubriant et, par extension, celui de Saint- 
Denys Garneau, représentent une fuite par rapport aux problèmes immédiats du monde. Ce que le poète de Sainte-Catherine apprécie chez l'auteur de la Brière, c'est le "mystique de la douleur (...) rehaussée à l'ordre spirituel $(. . .)^{\prime \prime 7}$. "l'expérience soufferte pas à pas par une âme en quête d'un support, (...) le témoignage de Saint Augustin sur la nouvelle lumière qu'il a trouvée au fond de lui-mème, (...) la confusion qui l'empêche d'assumer toute la réalité", le "refus de la matière"s. Chateaubriant croit à la grâce et à la charité pour dépasser les problèmes de ce monde. C'est une idée que partage Saint-Denys Garneau. Ferron, lui, s'élève contre l'obscure transcendance de Saint-Denys Garneau, contre le refus du monde de la matière, qui implique un refus de la réalité sociale. Quant a "Sieur Alphonse de tralala", Ferron l'accuse non seulement d'être un mystique incompréhensible, mais aussi d'être totalitaire: le "vieux bouc, chapelet dans sa poche et scapulaire au cou (...) finira nazi pour la plus grande gioire de Dieu (p. 176)"10.

En dernière analyse, le Saint-Denys Garneau de Ferron se présente comme une version diluée et déviée de l'arrière-grand-père historien pour qui l'histoire comptait plus que les déchirements intérieurs:

Orphée était ce fils de famille, ce grand garçon dérisoire qui se plaignait de ne pas retrouver la caisse à jouets de son enfance, celui-là même qui á écrit si ridiculement:

C'est eux qui m'ont tué

Sont tombés sur mon dos avec leurs armes

M'ont tué

Sont tombés sur mon coeur avec leur haine

M'ont tué

Sont tombés sur mes nerfs avec leurs cris.

Ve Ciel de Québec, p. 329).

Toutefois, dans certains passages, Ferron, toujours railleur et imprévisible, transforme son Orphée en un personnage qui a honte de s'être apitoyé sur lui-même, qui regrette sa "fausse dignite", sa "sotte gioire". Le poète proteste en effet contre les critiques qui ont fait de lui "le citoyen d'une terre qu'il a enrichie de sa souffrance" Ve Ciel de Québec, p. 330). Gráce aux transformations accomplies par le conteur, Orphée peut évoluer vers un certain dynamisme, vers une prise de conscience. Miraculeusement, il dit ne pas avoir voulu être le "faussaire" de François-Xavier Garneau. Et lorsqu'il fait son entrée dans le bordel de la rue Saint-Vallier, le lecteur est en droit de se demander si, à l'instar de Frank-Anacharcis du même roman, le poète de l' "intimisme" a définitivement trouvé, grâce à la putain, une conscience sociale. Mais Orphée n'arrive pas à se débarrasser de son intimisme destructeur. A la fin du roman, il redevient un poète aliéné. Après avoir quitté le bordel, il essaie de nous prouver que tout était inévitable: son désespoir, son manque d'espace, de saisons et de couleurs: "(...) un espace doré navant sans couleur, du pays funeste qui me verra me survivre malgré moi, hors de toute saison, vivant mais déjả mort" (p. 384). Et Monseigneur Camille, au nom de Ferron lui-même, de protester: "Non! non! il n'y a pas de destin en pays québécois où nous grouillons pêle-méle comme une portée de chiens" (p. 385)! II ne faut pas subir son sort, raisonne le prélat éclairé. II faut le changer, imposer l'"espace", la vie et la "couleur" de la collectivité. Durant sa dernière descente en enfer, l'Orphée ferronien a 
encore une fois plié devant son désespoir. Et pourtant, pour retrouver son amie Eurydice, il n'àvait qu'à ne pas se retourner vers elle avant de regagner la cuisine du bordel (même consigñe que dans la mythologie). Ėurydice et Orphée auraient pu ainsi participer à "l'extraordinaire lumière de notre pays à la fin de l'hiver, ( (... a) la blancheur radieuse de notre pays qui fuit de toutes parts et se-disperse sous le vol noir des corneilles, (...à) la neige (qui) fond déjà et (qui) comme nous remonte peu a peu la terre maternelle" (p. 390). L'entrée d'Orphée dans le monde de tous les jours aurait pu correspondre a son entrée dans le pays québécois retrouvé. Eurydice, elle, veut faire l'amour. La poésie nombriliste ne l'intéresse pas. Elle veut qu'Orphée la prenne comme une putain sur le plancher du bordel. Mais Orphée a peur de la réalité charnelle. Le descendant de François-Xavier continue donc de fuir le réel, se prolongeant dans son univers d'introspection et d'ossements. Lâche, il regarde Eurydice avant de quitter le sous-sol. Son visage est laid et ensanglanté:

(...) je l'ai aperçue. Un côté de son visage avait été écrasé. L'oeil était sorti et pendait, retenu par le pédoncule, sur la boue sanguinolente. L'autre côté était de cire avec des reflets violacés, l'orbite profonde avec des larves entre les cils Ve Ciel de Québec, p. 3921.

Saint-Denys Garneau est redevenu lui-méme, se complaisant, se disséquant dans "la mort grandissante".

Dans l'oeuvre caricaturale de Ferron, Anne Hébert est associée à son célèbre cousin (transformé, par association indirecte, en propre fils d'Anne Hébert):

Tu ne te nommes pas Saint-Denys Garneau!

Tu n'es pas le fils de Madame Anne Hébert!

Jean Goupil, tu n'es rien!

Va Chaise du marechal ferrant, p. 165)

Une autre Anne Hébert, celle de I'Impromptu des deux chiens, s'avère l'"enfant choyée" de la Société Royale, la dame "trop bien née (qui donne) grâce ainsi aux miteux de la haute ville de Québec... quel décor! Quel ensemble!" L'auteur des Historiettes, décrivant le lancement d'un livre de l'"abbé Pagé" sur Anne Hébert, s'égaie dans des charges contre l'auteur de "La fille maigre" que l'on ne peut comprendre sans se référer à la Contre-Réforme. Lorsque, dans le Torrent, la mère dit à son fils "Le monde n'est pas beau, il ne faut pas $y$ toucher," elle se serait exprimée "comme une nonne manqúée. Sainte-Thérèse ne parlait pas autrement" (p. 170-171). Pourquoi toutes ces diatribes? Sans doute parce que la première poésie d'Anne Hébert, aliénante, imprégnée de complexes catholiques, renferme la même sorte de déviation par rapport aux problèmes sociaux et patriotiques que la poésie de Saint-Denys Garneau ${ }^{12}$. Dans une scène tout à fait cocasse du Ciel de Québec, "Mademoiselle Hébert" pleurniche, refuse de se faire "striptiseuse" Iréférence humoristique au thème de la découverte du corps dans la poésie d'Anne Hébert) et affiche une aversion exceptionnelle pour la ceinture fléchée.

Après Saint-Denys Garneau et Anne Hêbert, c'est Jean Le Moyne qui joue le rôle le plus important d" "écrivain-personnage". "Grand jeune homme impulsif, épris de religion" Ve Ciel de Québec, p. 209), il se réfugie dans sa "brûlure profonde", ne 
boit pas, ne fume pas, ne joue pas aux cartes, se contentant d'honorer Dieu et d'exprimer en prose ce que Saint-Denys Garneau a traduit en poésie ${ }^{13}$. Cherchant à se réconcilier avec la cosmologie divine, le Le Moyne du "ciel ferronien" joue au janséniste: anges et démons piaillent autour de lui: "Janséniste! Janséniste! Jansénistel" (p. 248).

Une relecture de Convergences indique clairement les "divergences" entre Ferron et Jean Le Moyne. Le fil conducteur du recueil, c'est la "considération théologale ${ }^{\prime 14}$, considération évangélique inspirée du père de Jean Le Moyne qui refusait toute littérature "abstraite d'un contexte religieux"15. A l'encontre de Ferron, Jean Le Moyne admire la beauté de la dépossession chez Anne Hébert et fait l'éloge de Saint-Denys Garneau, inventoriant les transformations poétiques du dualisme, des amours empêchées et de la névrose du poète. Il le dit même "exemplaire en se niant" ${ }^{\prime 16}$, victime admirable d'une société dualiste. Voici donc Jean Le Moyne, loin de toute considération esthétique, qui loue Saint-Denys Garneau pour son exemplarité de martyr, alors que Ferron, aussi éloigné de la valeur poétique que Le Moyne, accuse le poète de lâcheté nationale! Cette incompatibilité idéologique s'accentue encore lorsque Jean Le Moyne approuve l'anti-nationalisme du poète de Sainte-Catherine. Colla borateur intime de Trudeau dont il a rédigé plusieurs discours d'ordre culturel, Jean Le Moyne a même osé prétendre que le nationalisme et l'indépendance mèneront à une tyrannie à la Franco: "(...) nous ne manquons pas d'aspirants-Franco conformes au modèle - taille comprise - et on frérnit à la pensée de ce qui arriverait à un peuple canadien-français constitué en Ėtat indépendant"17. Or, l'oeuvre de Ferron vise pré cisément à l'édification d'un pays indépendant, où le nationalisme lou plutôt le patriotismel, enraciné dans les événements de 1837, signifierait, non pas la tyrannie, mais une forme de social-démocratie. Jean Le Moyne appelle le nationalisme "réflexes sentimentaux", survivances ataviques relevant directement de la sauvagerie et compliquées d'orgueil"18, instincts irrationnels, xénophobes et rétrogrades. Caricaturiste hors de pair, Ferron riposte en transformant son Le Moyne en enfant de choeur qui sacre et dont l'encensoir serait la lanterne du "CN Raillerouillé limitée" Ve Ciel de Québec, p. 240). L'attitude anti-nationaliste de Jean Le Moyne évoque la "traque" confédérale, "voie" qui chez Ferron mène directement à l'assimilation, ou comme le dit si éloquemment le conteur du pays incertain, aux "vaches mortes du canyon".

Chaque fois que Ferron fait la satire d'un écrivain québécois, il s'agit presque toujours de quelqu'un qui ne s'engage pas socialement dans le pays, ou qui a une vision abstraite et mystique de la littérature. Claude Péloquin, "quémandeur de Christian Science" (/'Amélanchier), est associé à un évangéliste, car Pélo n'est plus engagé comme dans le temps de sa phrase retentissante: "Vous n'êtes pas tannés de mourir bande de caves!" II ressemblerait à d'autres qui ont suivi le mème cheminement, à Jacques Renaud et a Paul Chamberland préférant les voyages intérieurs et le nirvâna aux "cassés" et aux "afficheurs qui hurient", premières préoccupations mystérieusement abandonnes.

François Hertel représente ce que Ferron appelle la philosophie inconséquente de la "Blague", juxtaposant dans la Barbe de François Hertel, fauteuil episcopal et portrait de Sartre. L'ancien père Hertel est passé par le "teilhardisme" avant de devenir athée, ne retenant que la beauté mythologique et poétique du christianisme, s'inspirant pour le reste de l'exaltation du moi et du surhomme de Barrès. Ferron, égalitaire 
concaincu qui ne peut supporter le culte du imoi, transforme Hertel en un bouc inconséquent.

Paul Toupin incarne l'écrivain "apatride". Le Toupin des Roses sauvages pró fère les académies et les pays lointains aux réalités immédiates décrites par le "sympa thique Gratien Gélinas". Dramaturge et essayiste, Paul Toupin, bien que "doué pour l'art épistolaire", aurait été "débauche" par Montherlant ${ }^{19}, c^{\prime}$ est-ł̀-dire "converti" aux drames classiques, à l'admiration sans borne de la dignité espagnole. "En faveur auprès de Duplessis" (/"Exécution de Maski), il aurait ignoré les problèmes d'ici au profit de ceux de César (voir sa pièce Brutus) et des Bretons (voir le Mensonge). Il croit à un theâtre de fauteuil où la langue, purifiée et condensée, compte plus que l'acte théatral, alors que Ferron insiste sur le fait que le théâtre est, ou devrait être, une communica tion directe et spontanée entre spectateurs et dramaturge. Récupéré par Ferron, Paul Toupin se mue en un seigneur ambitieux (Nella Mariem) qui trahit son enfance et ses origines au nom de l'argent, monnaie du diable. II aurait fallu, raille l'Alber Millaire de I'Impromptu des deux chiens, envoyer Toupin chez les Bouroubourous d'Afrique.

Dans un domaine lié étroitement à la littérature québécoise, Ferron consacre de longues pages à "dégonfler le ballon" des Automatistes, "chevaux de parade" éloignés de la réalité d'ici (la Mort de Monsieur Borcuas/. Ferron qualifie Paul-Émile Borduas d" "habilleur académique", de "chérubin" parmi les "séraphins" surréalistes, d'homme qui théorise trop et qui n'est exemplaire que par son enseignement efficace et humanitaire (Historiettes). Ferron a néanmoins appris à aimer ce "petit homme honnête", poli, muni d'un dentier génant. Cependant il place Borduas, avec Orphée et Jean Le Moyne, dans I'"enfer des indécis", la couleur étant un peu à l'Automatiste ce que I'"intimisme" et l'espace étaient a Saint-Denys Garneau: une fuite face à la réalité sociale ${ }^{20}$. Dans le Ciel de Québec, le romancier affirme que Borduas a été victime d'une "boursouflure de renommée" fondée sur la "légende" de l'ostracisme dont il a souffert. Le Borduas ferronien voit clair en lui-même, refuse la "rengaine du génie méconnu par les siens et que son pays fait mourir" (p. 240). Dans un moment de clairvoyance, il jette bas "toutes les fausses valeurs qui l'ont mystifie" (p. 245). Selon Ferron, les peurs énumérées par les auteurs du Refus globa/ témoigneraient de la "niaisorie" de l'époque et ne constitueraient donc pas une découverte géniale: surmonter les préjugés, par exemple, c'était déjà un "idéal de Papineau": surmonter la pauvreté, c'est un idéal normal et évident; ne pas avọir peur de l'ordre établi, les Chénier et autres Patriotes le proclamaient à haute voix; surmonter la peur du surrationnel, le folklore et la mythologie du Québec en témoignent depuis trois cents ans ${ }^{21}$.

L'automatiste Claude Gauvreau, le vrai père "putatif" et "nourricier" de l'automatisme 22 , s'apparente, par ses paroles vagues et surréalistes, a Borduas. II descend en enfer voir Orphée et lui faire part de "l'embarras où le mot le tenait, le mot en soi, libéré du dictionnaire et de toute signification" Ve Ciel de Québec, p. 2331. Gauvreau représente le type de l'écrivain absolu dont les poèmes étaient "insensés et similiparnassiens"'23. Et Ferron d'insinuer que si le poète automatiste avait eu le sens de la collectivité, il ne se serait peut-être pas suicidé:

II n'avait pas les mèmes idées que (moi) sur la langue, greffe d'un sens com- 
mun dans le cerveau de chacun, qui permet à chacun d'être d'un pays, de faire partie d'un peuple ${ }^{24}$.

\section{Les antagonistes 6trangers}

Il existe dans l'oeuvre de Ferron une série de références à des écrivains étrangers qui deviennent les symboles d'une certaine "mauvaise conduite" de l'homme vivant en société. Dans les Roses sauvages, le romancier condamne Colette Baudoche, roman "ridicule" et "illisible" (p. 14), "fallacieuse elégance sur un grossier fatras" (p. 77). Le nationalisme mystique de Barrès, où s'entremélent les cultes de la patrie, des soldats et des morts, s'oppose au patriotisme ferronien ancré dans le réel et le quotidien, patrio tisme issu d'une situation historique qui n'a rien à voir avec le culte du moi, l'égoisme méthodique, et même la haine des étrangers si évidente dans l'oeuvre de Barrès. Chez Ferron, l'étranger - celui qui respecte les droits de la majorité francophone - est au contraire un véritable héros.

La Chaise du maróchal ferrant fait découvrir un Stendhal qui incarne, tout comme Barrès, l'égoïsme dévastateur. Chez Ferron, Stendhal, qui avait beaucoup influencé Barrès d'ailleurs, est rangé đu cóté des "gagnestères" et des "boulés". Do Boulé, contrebandier à l'emploi du diable capițaliste, "boulé dans le même sens que dans la Chartreuse de Parme" (p. 114), exploite systématiquement les petites gens. Pourquoi Stendhal fait-il penser aux "boulés"? Sans doute parce qu'il recrée un monde où l'on tue et empoisonne, qu'il s'agisse de Parme ou de la çour de Borgia. Grâce à la récupéra tion caricaturale et fantaisiste si typique de Ferron, Beyle fait partie de la mafia montréalaise - là où régne le culte du "héros" criminel - et encourage le favoritisme politique: Jean Goupil, qui ne sait ni lire ni écrire, utilise des moyens stendhaliens dignes "de la belle et noble Mathilde de la Mole" (p. 127) pour entrer au Sénat. Ainsi Ferron rattache-t-il l'ambition politique de Monsieur le Sénateur Goupil à cette gloire si dangereuse, rouge dans l'armée, noire dans l'Église, immortalisé par l'auteur du Rouge et le Noir. Saint-Simon joue un rôle semblable: une amie du sénateur Lesage, futur "duc", lit passionnément Saint-Simon et réve de noblesse. Tout comme Stendhal le duc de Saint-Simon évoque les "barons", les "ducs" et les "petits sénateurs" ferroniens, tous ceux qui jouissent des privileges de leurs titres et qui jouent au "Papa Boss de l'industrie".

Deux ecrivains canadiens-anglais reflètent eux aussi différentes formes de répression et d'abus. En général, les Canadiens anglais incarnent le paternalisme. II y a, par exemple, "Sieur MacLennan" qui encourage le prolongement des "Two solitudes", qui parle de "la plus étrange des complicité, two solitudes, may be, mais rien de tragique, car elles seraient voulues et consenties" Ve Ciel de Québec, p. 3511. Mais Ferron ne les trouve pas "consenties", et lorsque, dans un autre roman, François empoisonne un certain Frank assimilateur, le poison constitue une sorte de réponse aux Two Solitudes de Hugh MacLennan:

(Hugh), ami de Frank, incurable imbécile qui n'a jamais rien réussi que ce titre (Two Solitudes). II se situe à un moment de l'histoire où ces deux solitudes, tentative d'évitement qui a duré deux siècles, semblable a celle de deux bandes de singes hurleurs parvenant à partager un mème territoire de chasse, prévenus 
par leurs cris, en ne se rencontrant jamais, deviennent incongrues par l'extension naturelle du français rejoignant après 1960 toutes ses possibilités, cessant dès lors d'être au Québec un idiome provincial. (Appendice aux Confitures de coings ou le congédiement de Frank Campbell, p. 264).

L'écrivain Northrop Frye, ancien Québécois, Anglais "de nation et de profession qui préfère l'anatomie de la critique et de l'Université de Toronto à l'anatomie du Québec"25, subit une transformation pour le moins inusitée en lapin! Monsieur Allyre-Alexander Northrop, au nez rond et à la peau rose, passe son temps à tirer sa montre-boussole de la pochette de son gilet et à s'exclamer "ouhonnedeurfouledé" V'Amélanchier, p. 11). Squatter anglais dont Ferron ne reconnaît pas le droit de propriété en terre québécoise, Northrop se réfugie dans un terrier en attendant que les chênes du bois québécois soient assez grands "pour être réquisitionnés par la marine de Sa Majesté la reine Victoria" (p. 92). Mais la jeune Tinamer résiste au "déménagement" de son bois, l'entreprise de reboisement suscite "un fier ennemi en la personne de Léon de Portanqueu, esquire, fin causeur et fils de cultivateur" (p. 92) qui lance des cailloux à Northrop. Inquiet de l'avenir du pays, - "Un pays qui ne se nourrit pas lui-même n'est pas un pays" (p. 101) - Léon condamne Northrop le lapin, symbole des spéculateurs et des vendeurs de terres québécoises.

\section{Les protagonistes québécois}

A l'encontre des Northrop et MacLennan antipathiques, Ferron incorpore dans son oeuvre de nombreux auteurs qui sont présentés sous un angle totalement différent: en véritables héros. Chez les Québécois, il y a cependant peu d'écrivains qui répondent à un ou à plusieurs des critères ferroniens: faire de l'histoire "corrective" (être patriote dans le sens de 1837); faire de la petite histoire en nommant la flore, la faune et les petites gens du pays; combattre les thésauriseurs et les profiteurs urbains.

Ferron considère que Nelligan est un des plus grands poètes du pays. Une telle attitude a de quoi surprendre, car, en règle générale, Ferron trouve que toute poésie qui décrit un drame personnel est défaitiste, constitue une lâcheté lla dépossession de Saint-Denys Garneau; l'aliénation de Nelligan). Mais Nelligan n'est pas de la même famille qu'Orphé:

Nelligan (...) fut un héros au sens irlandais du mot. Je m'explique. Il s'agit d'engagement. À une époque où l'on niait l'existence d'une littérature nationale, où le pays était conçu comme l'enfer de l'homme de lettres, je crois que Nelligan fut un écrivain engagé, le premier que nous ayons eu. Adolescent, il se donne tout entier à la poésie et durant près de trois ans conçoit et exécute son oeuvre envers et contre tous. Envers sa mère, née Hudon, pauvre femme effacée qui ne jouait du piano que pour montrer qu'elle avait reçu une éducation distinguée; contre son père, Irlandais déculturé, ni Français ni Anglais, robot des Amériques; contre l'École littéraire de Montréal où la poésie n'était qu'un prétexte a confré rie, un subterfuge pour s'infatuer chacun de soi, voire pour s'embourgeoiser; une oeuvre hautement significative qui annonce clairement qu'il s'y épuise et qu'il en sortira dévitalisét $e^{26}$. 
Nelligan représente donc le poète qui croit à une littérature nationale ${ }^{27}$. II est également associé aux nombreux Irlandais enquébécoisés dans l'oeuvre de Ferron et aux ennemis des matérialistes, "robots des Amériques".

Au vingtième siècle, Ferron a une prédilection pour les auteurs populistes. Orphée voyant temporairement clair en lui-mème et condamnant une poésie évasive, vante les mérites de deux poètes: Jean Narrache, interprète du peuple, critique d'une bourgeoisie avare; Alfred DesRochers, chantre de la "communion" avec le sol québécois. Un autre auteur qui ressemble à Jean Narrache et à Desrochers apparait dans le SaintElias: Clément Marchand. Né dans le territoire même du roman, à Sainte-Genevièvede-Bastien, ce poète, inspiré par la misère trifluvienne, condamne l'usine qui fait courber les prolétaires. Il découvre son vrai pays à travers le "récitatif" de la "bonne souvenance" (dans son recueil les Soirs rouges), sorte de mémoire héréditaire qui pousse les hommes à refaire le milieu urbain.

C'est aussi l'amour du passé, de la tradition orale et du folklore, qui a poussé Ferron à citer Marius Barbeau: "La gloire et la beauté sont patientes. Kamalmouk, toi qui sais qu'elles ont été, apprends qu'elles reviendront". Ve Saint-Elias, p. 9). Anthropologue, folkloriste, spécialiste de la culture amérindienne et des traditions orales, l'auteur du Rêve de Kamalmouk étudie la vie des Tsimsyans de la Côte Nord-Ouest et condamne les responsables du génocide amérindien: le "missionnaire avec son gros livre, le chercheur d'or (...), le constable d'armes"28. La tragédie de Kamalmouk, chef humilié du clan des Loups, esclave du maittre blanc, rappelle le drame de Joseph-à-Moïse-à Chrétien du Ciel de Québec. Mais Kamalmouk, contrairement à Joseph, rejette son nom de colonisé (Jim) et tâche d'éveiller, d"'amérindianiser", les George, Alexis, Freddé et Thom du clan. Le chef est finalement abattu par l'agent Billy Green, mais la femme de Kamalmouk, dans un discours violent sur la perte de l'identité nationale, continue la besogne de son mari. Ainsi cette fière Tsimsyanne, Rayon-de-Soleil, surnommée Fanny par les blancs, ressemble-t-elle à la sage capitainesse des Chiquettes du Ciel de Québec.

Le frère Marie-Victorin occupe une place privilégiée dans le réseau des écrivains québécois sympathiques. Comme tout bon religieux ferronien, Marie-Victorin est "ignorantin". Le frère Thadéus du Salut de I"Irlande prétend que les ignorantins s'appellent ainsi parce que les autres ordres, plus élitistes, les méprisent. Ferron donne ainsi une explication toute personnelle à l'origine des frères ignorantins, nom qu'avaient pris par humilité, les religieux de Saint-Jean-de-Dieu. Thadéus est fier du sobriquet "ignorantin"... "Par ce sobriquet nous participions a l'humiliation des classes populaires" (p. 186). Et c'est Marie-Victorin qui s'avère l'ignorantin par excellence. L'auteur de la Flore Laurentienne aimait ses plantes, et l'amour dont il est question ici, c'est "la grande leçon de l'évangile. Aimer, aimer de n'importe quelle façon, aimer n'importe qui, $n$ 'importe quoi... Marie-Victorin, lui, aimait la terre, la sale terre des champs, celle qui noircit les mains et porte la patrie" Ve Salut de l'Irlande, p. 88). Le frère MarieVictorin, contrairement à la plupart de ses frères en religion crés par Ferron, ne fait pas de politique mesquine. Parce qu'il n'avait jamais été tout à fait un "bleu", certains cercles gouvernementaux et ecclésiastiques le condamnaient sans cesse. Mais il est mort content d'être "ignorantin" et amoureux d'une "petite fougère" de SaintHyacinthe ${ }^{29}$. 
Ferron est reconnaissant envers les hommes d'Église qui se sont consa. crés à la sauvegarde du pays. II nous fait découvrir un Marie-Victorin qui a refusé de devenir prêtre pour rester au service du pays à nommer. C'est d'ailleurs chez Marie-Victorin que Ferron a trouvé l'épisode des spéculateurs fonciers traversant le pont pour ensuite faire de Longueuil un bourbier infect. En effet, une nouvelle de Marie-Victorin est à l'origine du Salut de l'Irlande: "Ne vends pas la terre", portrait du vieil habitant longueuillois, Felix Delage, qui refuse de vendre sa terre à l'agent d'immeubles Mister Stevenson, malgré les vingt-cinq mille dollars offerts comptant. Les spéculateurs répugnaiẹnt autant à Marie-Victorin qu'à Ferron:

La folie de la spéculation immobilière, après avoir ravagé l'île de Montréal, débordait à présent sur la rive sud, submergeait les abords du vieux Longueuil et s'avançait dans la campagne. Comme des malsains champignons, surgissaient au milieu des champs, hideusement badigeonnées, les petites cabanes carrées des agents d'immeubles ${ }^{30}$.

\section{Les protagonistes étrengers}

Parmi les auteurs français transformés en "personnages" sympathiques, Louis Hémon est le plus fascinant. Dès la Téte du roi (1963), on apprend que le refrain pré monitoire de Louis Hémon s'applique toujours: des étrangers sont venus, ils ont pris presque tout le pouvoir, presque tout l'argent. Dans le Ciel de Québec, le Révérend Dugald Scot, qui n'aime pas les Canadiens français, se fie à la conclusion de Maria Chapdelaine: "Au pays de Québec rien ne doit mourir et rien ne doit changer" (p. 350), ni pour les Ėcossais privilégiés, ni pour les Québécois soumis. Mais l'abbé Surprenant n'est pas du tout d'accord avec son confrère protestant. Influencé par un autre roman 'de Louis Hémon, Monsieur Ripois et la Némésis, l'abbé travaille à améliorer le sort des non nantis. II se souvient de cet Amédée Ripois qui déplore la misère des chômeurs perdus dans la mêlée, errant dans Soho, "pourchassant d'un métier à l'autre des choses essentielles: un lit et du pain" ${ }^{\prime 31}$. Pour Surprenant, l'"exposition permanente de la vie nocturne à Londres"' rappelle les tumultes laborieux de la foule montréalaise. II comprend la notion des "barbares" lassociés, dans Monsieur Ripois, aux policiers corrompus, dans Maria Chapdelaine, aux étrangers capitalistesl, car ce sont deux bandes de "barbares" caricaturées sans cesse dans l'oeuvre de Ferron.

Dans la préface de Colin-Maillard, Ferron décrit un Louis Hémon en désaccord avec son frère Félix mort après s'être battu en Chine contre les Boxers "justement révoltés" ${ }^{32}$. Hémon a traversé la Manche pour se dissocier de la politique coloniale de la France. II a renié la philosophie colonialiste, la gloire cornélienne de son père (le père de Louis Hémon, inspecteur de l'Académie de Paris, a écrit une préface pour Don Sanche d Aragon, publiée en 1896/33. II s'est associé aux Irlandais attaquant l'impérialisme britannique:

Breton. Celte comme les Irlandais, Hémon n'était qu'un particulier hostile aux Etats qui écrasaient les peuples et qui, même de nos jours, n'ont pas fini de les ecraser (...) II quittera pour toujours la France, dont il pouvait désavouer la politique coloniale, le chauvinisme, le complexe de supériorite ${ }^{34}$. 
Les liens idéologiquies èntre Ferron et Louis Hémon sont tellement étroits que les personnages des deux auteurs ont la curieuse habitude de se mélanger. Le Mike O'Brady de Colin-Maillard aurait pu se nommer "Michel Bradette de Saint-Malachie dans la rue Colonial à Montréal'35. En l'espace de quelques lignes, Mike O'Brady se métamorphose en un Irlandais ferronien francisé, sorte de frère de Connie Haffigan conscient du fait que la lutte contre le colonialisme, pour un Irlandais québécois, doit se faire à côté des Québécois francophones.

Si Louis Hémon s'intégre dans l'oeuvre de Ferron comme l'ami des petites gens et des petits peuples, Molière apparait comme le dénonciateur par excellence des profiteurs de tout genre. Le valet Jasmin, sorte de Sganarelle ferronien, est inspiré de Molière, chez qui le théâtre reflète, par ses machinations et ses diableries, le ridicule de la comedie humaine (/'Ogre), ridicule qui est rappelé par des noms de personnages qui sont communs aux deux écrivains (Agnès: I'École des femmes, le Dodu; Dorante: la Critique de I'École des femmes, le Licou; Élise: I'Avare, Tante Elise ou le Prix de I'amour; le Commandeur: Don Juan, le Cheval de Don Juan).

Ferron se sent trés près du Misanthrope, incarnation de l'être solitaire, de l'“orphelin" que nous sommes tous. L'Alceste à l'âme si noble revu par Ferron s'en prend aux mensonges mondains, à la fausse sentimentalité, à la galanterie, a la coquetterie et aux procès injustes:" (...) par son franc-parler, ses frondes, ses procès qu'il perd avec jubilation, content de pouvoir narguer la société de son mépris, ...Alceste se prouve ainsi qu'il est libre, son propre souverain"36. Molière, c'est l'esprit frondeur, narguant, en s'amusant, les hypocrites:

Dans un monde factice où le masque prévient le visage, où le rôle résume I'homme, comme des mouches dans le miel on s'agglutine aux apparences; le masque se colle au visage, le rôle marque l'homme; on vit comme au théatre, oubliant le reste du monde, sur une scène exiguè. Ve Cheval de Don Juan, p. 205).

Théâtre, théâtral, mise en scène, carnaval, masque, cirque, mascarade, marionnette, festival, foire, cérémonie, figurants, figuration, comédiens, voilà les mots auxquels Ferron a recours lorsqu'il traite des innombrables conspirations qui animent son oeuvre: ecclé siastiques, homme politiques, capitalistes, auteurs et médecins aveuglés par la pré séance. A l'encontre du déploiement théâtral capitaliste, Ferron propose le "théâtre intime" de réflexions personnelles aiguisant la conscience sociale et nationale (I'Amélanchier). La société étant un plateau d'illusions, une "mauvaise comédie" (I'Ogrel, l'oeuvre littéraire préconisée par Ferron met en scène, en les grossissant, les illusions de la vie.

Puisque Ferron est avant tout un conteur, il est normal de voir apparaitre dans son oeuvre de nombreuses références à d'autres conteurs. Dans I'Amélanchier, par exemple, Tinamer s'inspire de l'épopée chevaleresque de Jacques Cazotte, Ollivier. Elle $y$ a puisé toute une ambiance, reprenant, parfois textuellement, l'épisode d'Enguerrand et de Strigilline ${ }^{37}$. Bien sûr, des transformations importantes ont lieu: le perroquet devient la bécasse du Canada, les truffes et le vin se transforment en mandarines et en pepsi, le président en Léon, la fée en Etna, et surtout, les six oiseaux en six poules endoctrinées par le Papa Boss. C'est de cette façon que le féerique d'Ollivier prend la forme d'une fable qui dénonce les dangers du capitalisme. 
Le conte de fée engagé, forme privilégiée par laquelle Ferron exprime ses idées, dissimule à peine ses références à Charles Perrault. Dans Papa Boss, l'"accotée" espère se marier, connaître le bonheur, être la "fernme qui éclaire sa maison bien ornée comme le soleil se lève dans les hauteurs du Seigneur" (p. 120). Mais un peu comme dans les contes de fée (Cendrillon), le mari n'arrive pas à mettre son soulier qui est trop petit: le mariage n'aura pas lieu. Dans les Grands Soleils, Cendrillon est encore une fois sous-jacent: Ferron craint que les "grands soleils", symbole du patriotisme, ne "deviennent citrouilles", que le "carrosse du pays" imaginé par Mithridate ne se trans forme en citrouille, marquant ainsi la fin du réve de Chénier. Ailleurs, nous apprenons qu'en amour tout est jeu: la femme est une souris, l'homme un chat botté allant d'une chambre à coucher à une autre (/e Licou/; le petit chaperon rouge est la victime d'un viol, ou presque, l'homme étant un loup pour la femme l"le petit chaperon rouge". Contes anglaisl. Dans l'immense fable ferronienne, les chats bottés sont à l'occasion des "chats percés" (des pauvres) qui souffrent dans les Basses-villes (le Ciel de Qué$b e c$ ), des matous qui pissent sur les profiteurs et sur I'humanité mauvaise (la Nuit).

Le conteur Lewis Carroll est un écrivain que Ferron aime beaucoup fréquenter. II a passé de longues et agréables heures à lire à sa fille Alice au pays des merveilles. Le célèbre récit de Carroll ressemble énormément à celui de Tinamer. Alice rencontre un Lapin Blanc aux yeux roses habillé en redingote avec chapeau melon et gants blancs de chevreau. Le Monsieur Northrop de Tinamer a le même genre que ce "gentleman" anglais transformé en lapin. Le Lapin Blanc aux yeux roses possède une montre qui $n^{\prime}$ indique que le jour du mois, jamais l'heure. Les personnages du pays des merveilles n'ont donc pas besoin d'orientation temporelle.

Tinamer et Alice sont victimes d'un même phénomène: elles rétrécissent, la petite héroïne britannique sous l'effet de champignons, de gâteaux et de breuvages, Tinamer, grảce à une pommade spécifique. L'énorme pied droit d'Alice lle rétrécissement ne se fait pas toujours d'une façon égale) s'appelle dérisoirement "esquire", tout comme le père orgueilleux de Tinamer. Alice et Tinamer partagent encore autre chose: elles ont des animaux qui semblent appartenir au bon côté des choses (le merveilleux), qui combattent l'injustice (pour Alice, il s'agit surtout de "Cheshire Cat". Dinah; pour Tinamer, du merle-chat).

Derrière la fantaisie féerique et les jeux de mots de Lewis Carroll se cache, comme chez Ferron, toute une satire sociale. La Dame de Coeur, qui crie constamment "Off with your head!" ("Qu'on le décapite!" ${ }^{38}$, incarne la tyrannie du pouvoir. De même l'existence antérieure de Northrop est liée à une certaine Duchesse qui voulait lui couper la tête. Et lẹ bébé-cobaye, vrai cochon d'Inde, qui symbolise le malheur des enfants maltraités, renvoie à la satire du Mont-Thabor dans I'Amélanchier.

La ressemblance la plus frappante entre Alice au pays des merveilles et l'Amélanchier réside sans doute dans la nécessité proclamée, en littérature, d'une vision enfantine à la fois instructive et divertissante pour aider a corriger l'horreur des asiles, des "reines au coeur méchant" et des procès absurdes. Pour Ferron, le conte est l'occa sion de remettre en question l'ordre établi. Tel est le cas, par exemple, des Mille et une nuits, où le lecteur découvre le "salut du monde par la fantaisie: voilà une Bible qui vaut bien l'autre" ${ }^{\prime 39}$. Le Manchot de l'Ogre va mème jusqu'à affirmer que s'il 
"n'avait pas l'Arabie dans la tôte, il serait complètement perdu" (0. 49). Et l'Arabie, c'est une des plus belles fables qui révèlent le ridicule de la condition humaine. Dans l'Ogre, le ridicule apparaít à travers le "conte arabe" du Manchot qui démontre que l'amour n'est que trop souvent une lutte absurde entre captif et captive. L'Arabie est partout, dans le rang de Fontarabie où s'effectuent de merveilleuses transformations d'animaux (dans "Bêtes et maris", Contes anglais), dans l'Alcazar, boîte mauresque où se réunissent policiers, putains et bandits (la Nuit), dans les écoles où les professeurs parlent en latin moliéresque et bafouillent "Ali baba" ("Mélie et le boeuf", Contes du pays incertain), dans le personnage de la petite Tinamer, aussi légendaire au Québec que la grande reine de Saba (I'Amélanchier).

Lewis Carroll n'est pas le seul auteur britannique à jouer un rôle déterminant dans l'oeuvre de Ferron. Frank-Anacharcis, se sentant coupable de son rôle de missionnaire, cite son ecrivain anglais préféré, Samuel Butler: "(...) il ne faut pas parler latin a des bouviers qui viennent de perdre leurs boeuts" (p. 142), phrase qui signifie, dans le contexte du Ciel de Québec, qu'il ne faut pas, au nom de la religion, imposer sa langue aux autres, qu'il ne faut pas déranger le mode de vie d'un peuple (les besoins des Amérindiens).

C'est dans le Saint-Elias que les références a Butler prennent toute leur signification. Ferron y loue les mérites "d'un Anglais du nom de Samuel Butler qui a écrit un livre intitulé Erewhon, où un peuple heureux garde au musée toutes les machines qu'il a inventées et dont il a été assez sage pour ne pas se servir. Ce livre aurait été écrit à Montréal" (p. 158). Butler a connu, comme l'autre écrivain londonien que Ferron affectionne tant, Charles Dickens, l'industrialisation inhumaine de la GrandeBretagne. Il a brièvement joué le jeu du capitalisme à Montréal où il a essayé de faire fortune dans la "Canada Tanning Extract Company". Dégouté par la corruption des membres de la "Bank of Montreal", par les transactions malhonnêtes des financiers, Butler a écrit son Erewhon (il l'aurait écrit en Nouvelle-Zélande et non pas au Canada, comme le prétend Ferron), satire fantaisiste sur la machine qui contrôle l'homme, et sur la famille répressive qui empêche l'épanouissement de l'enfant. La parenté entre Ferron et Butler ne s'arrète pas là, car la religion des "Erewhonians" ressemble, avec les "High Ydgrunites" de la déesse Yodgrun et les "Low Ydgrunites" plus terre à terre, à la hiérarchie ecclésiastique tant décriée par l'auteur du Ciel de Québec. Samuel Butler fait tellement partie du Ciel de Québec que la mère de Frank-Anacharcis est censée I'avoir connu personnellement. Ferron aime ressusciter ses écrivains préférés, les pré senter à ses personnages. "Obligé de séjourner à Montréal, alors ville putain des chemins de fer, aujourd'hui des parkings et du pétrole sulfureux" Ve Saint-Elias, p. 181), le Samuel Butler de Ferron est le précurseur des critiques du déferlement urbain et de la destruction des ressources de la terre, par "cupidité, par leur gaspillage qu'ils nomment consommation" (p. 183).

L'équivalent américain de Samuel Butler est sans conteste Nathaniel Hawthorne. L'Ann Higgit des Roses sauvages reste possédée par la noblesse, par l'horreur, par le pessimisme ét par la "grandeur tragique" de Hawthorne, ce grand pessimiste réfractaire aux transcendantalistes et au mysticisme du panthéiste Emerson. Ferron mentionne plusieurs fois, directement ou indirectement, le romancier Nathaniel Hawthorne. Dans la Créance, nous apprenons que le père de Ferron se vantait de sa maison à 
cinq portes qui, comme la maison à sept pignons dans l'oeuvre de Hawthorne, indiquait la suffisance du propriétaire. Dans The House of the Seven Gables, le romancier de la Nouvelle-Angleterre centre l'action de son livre sur la maison des Pyncheon, ancienne demeure du colonel Pyncheon. Le colonel incarne cet "absurd delusion of family importance which has always characterized the Pyncheons" ${ }^{\prime 40}$. L'orme ances tral devant la maison pourrie ainsi que le portrait miroitant du colonel représentant ce que Ferron appelle le "préjugé aristocratique". En effet, Hawthorne fait la satire de l"'idle aristocracy", des juges qui envoient les innocents dans les asilés, des pasteurs vivant de favoritisme. Et comme Ferron, il utilise le merveilleux pour faire passer ses idees, "mingling the Marvellous as a slight, delicate, and evanescent flavour"41. Les roses des Pyncheon remontent au temps de l'ancètre exploiteur qui avait fait fortune dans les plantations. Symbole de l'éphémère continuité aristocratique, elles ressemblent aux roses sauvages de Ferron, symbole de l'ambition mal placée. De plus, la pers pective historique du romancier américain se rapproche de la vision de Léon de Portanqueu qui raconte l'histoire de la fondation de villages et de familles. Dans The Scarlet Letter, qu'Ann Higgit a également lu avec passion, Hawthorne s'attache à son appartenance familiale et nationale. Après avoir lu les documents du "Customs House" (poste de douane), un peu à la façon de Ferron recueillant les témoignages des Jésuites et des Ursulines, le romancier de Salem recrée l'époque des condamnations des sorciéres par les extrémistes puritains qui jugeaient et parfois exécutaient au nom des Saintes Ecritures. Hawthorne croit à la nécessité d'un "home-feeling" avec ses ancêtres:

The figure of the first ancestor invested by family tradition, with a dim and dusky candour, was present to my boyish imagination. It still haunts me, and induces a sort of home-feeling with the past ${ }^{42}$.

II existe dans l'oeuvre de Ferron, une unité fascinante. Les nombreux textes littéraires auxquels nous nous sommes référé, diversifiés par des situations et par des représentations différentes, apparentés par la pensée qui les anime, créent un vérita ble faisceau de personnages et de situations, signes sans cesse vivants d'une pensée sociale cohérente et claire, humanitaire et fraternelle, engagée et engageante. Les allusions aux écrivains d'ici et d'ailleurs forment un systeme signifiant qui sert de filtre aux idées sociales de l'auteur. Les écrivains du pays de Québec ont des règles à suivre pour devenir des "personnages" sympathiques. Grosso modo, ils doivent rester fidèles aux idées que Ferron avance sur l'histoire, sur le capitalisme, et sur la religion. Ils ne doivent surtout pas se complaire dans des métaphores d'aliénation, théoriser au lieu d'agir, être "intimistes" et non engagés. Ferron a une prédilection pour l'écrivain qui, conscient de la continuité historique de son pays, puisant à même le folklore et les légendes, faisant du féerique une façon de percevoir et de corriger la réalité, dénonce la farce moliéresque qu'est la vie et incite à l'action pour améliorer le sort de l'homme.

1. Jean Marcel, Jacques Ferron malgré Iui, p. 101-102.

2. Antiony Hamitton, Mémoires du comte de Gramont, éd. Rencontre, 1964, 336 p. 
3. Faute d'espace, nous ne discuterons pas des ecrivains qui ont influencé Ferron mais qui n'apparaissent pas dans l'oeuvre littéraire (entre autres, George Eliot et Charles Dickens), ni de toute une série d'écrivains épisodiques et somme toute mineurs dans le contexte de l'oeuvre ferronienne Nictor Barbeau, Nérée Beauchemin, Michel Bibaud, Robert Charbonneau, Léon Gérin, Alain Grandbois, Claude Hurtubise, Anatole Parenteau, Naïm Kattan, Pierre Vadeboncoeur; les critiques Gérard Bessette, Eva Kushner, Gilles Marcotte; les écrivains britanniques Daniel Defoe, Edward Young et Shakespeare; l'écrivain américain John Howard Griffin; les auteurs français André Breton, Cyrano de Bergerac, Gobineau, lonesco, Rotrou, Valéry, Vauvenargues; le romancier canadien-anglais Scott Symons; le Guatérnaltèque Asturias; le naturaliste suédois Pierre Kalm).

4. Voir la Relève, vol. III, nos 5 et 6, 1937.

5. Voir Paul Beaulieu, dans la Releve, vol. I, no 6 (1937), p. 153-164.

6. Voir Paul Dumas, dans la Relève, vol. I, no 8 (1935), p. 185-195.

7. Saint-Denys Garneau, "Alphonse de Chateaubriant", la Re/\&ve, vol. II, no 3 (1935), p. 76.

8. Saint-Denys Garneau, "Alphonse de Chateaubriant", la Relève, vol. II, no 6 (1936), p. 166.

9. Saint-Denys Garneau, "Alphonse de Chateaubriant", la Releve, vol. II, no 7 (1936), p. 210 et p. 213.

10. Au collège Brébeuf, Ferron a eu un professeur qui déclamait Chateaubriant "devant le SaintSacrement. (...) Chateaubriant prit bel et bien parti pour l'Allemagne de Monsieur Hitler après la défaite de la France, donc le plus bravement du monde. II aurait ́́té recueilli en Autriche, après la chute de Berlin, par une brave personne, Madarne Petzold, qui avec la complicité du saint curé Schmid va lui ouvrir sa maison. J'aime bien cette complicité du saint curé. Sans doute, avait-il appris qu'au Canada on déclamait du Alphonse de Chateaubriant" (Jacques Ferron, "Le mimétisme transcendental", dans Escarmouches, la longué passe, t. II, p. 192-193).

11. Jacques Ferron, IIImpromptu des deux chiens, dans Theatre II, Librairie D6om, 1975, p. 178.

12. Ferron ne prise pas du tout les "mises en bointes, chambres de bois, tombeaux, tous les procédés qui ont porté mademoiselle Anne Hébert à la Société Royale, ce palais des momies" (Jacques Ferron, Escarmouches, la longue passe, t. II, p. 96).

13. Convergences "a valu à son auteur, avant le Molson, tous les autres prix littéraires du Haut et du Bas-Canada. C'était un premier livre rempli d'une prose déja publiée çà et là dans les revues. Il a été tellement célébré, équivalent prosaïque des poèmes de feu Hector de SaintDenys-Garneau" (Jacques Ferron, Escarmouches, la longue passe, t. II, p. 122).

14. Jean Le Moyne, Convergences, HMH, 1962, p. 7.

15. Ibid., p. 17.

16. Ibid., p. 238.

17. Ibid., p. $50-51$.

18. Ibid., p. 35.

19. Jacques Ferron, I'mpromptu des deux chiens, dans Theatre II, Librairie Déom, 1975, p. 179. Dans ses articles, Ferron parle souvent de Toupin: "Notre littérature n'a pas fini d'accoucher des monstres. Par exemple le Brutus de Toupin. Que vient faire Brutus dans cette aftaire? Ce sera un crapaud qui bouffera tous nos petits césars" IJacques Ferron, Escarmouches, la longue passe, t. l, p. 59-60). Paul Toupin, "elève des Jésuites, certain de sa supériorité et quelque peu piqué qu' un petit homme sans diplôme, Gratien Gélinas, formé a la dure école, lui soufflàt une gloire à lui réservée" (Jacques Ferron, "Notre théatre" dans Escarmouches, la longue passe, t. l, p. 221.

20. Ferron apprecie cependant la "présentation" des tableaux de "Maitre Borduas", car Borduas "avait un talent d'ensemblier" (Jacques Ferron, Du fond de mon arrière cuisine, $\mathrm{p}$. 247). Mais pour I'auteur des Escarmouches, les Automatistes étaient "simplistes et livresques, causant de générosité, de mesquinerie, oubliant le principal, la structure qui nous a tenus depuis toujours à l'écart du monde, en dehors de I'histoire" (t. l, p. 173).

21. Voir Jacques Ferron, Escarmouches, la longue passe, t. II, p. 13, p. 177. 
22. Voir la Mort de Monsieur Borduas. Ferron prétend que Gauvreau aurait été le grand responsable des manifestes automatistes, qu'il écrivait alors que les autres Automatistes, "petite chapelle", vivaient d'actes volatiles et futiles IMousseau a brôlé un drap funébre pour se venger de l'Eglise; Barbeau criait sans cesse que le dadaisme était mort).

23. Jacques Ferron, Du fond de mon arrière-cuisine, p. 250.

24. Ibid., p. 275.

25. Entrevue avec Jaçques Ferron, Lettres québécoises, no 6 (avril-mai 1977), p. 38. Le professeur Frye, auteur de l'Anatomy of Criticism, spécialiste des mythes antiques, est né a Sherbrooke.

26. Jacques Ferron, "Littérature utilitaire et l'ecrivain engage", dans Escarmouches, la longue passe, t. II, p. 140-141.

27. "Nelligan n'est pas victime d'une réputation surfaite ni gonflée, comme c'est le cas de SaintDenys Garneau. Nelligan n'est pas un petit Baudelaire né en Amerique, n'est pas un châtré social. II fait des poèmes extraordinaires. Comparé à Saint-Denys Garneau, qui se disait d'un espace irréel et de nulle part, Emile Nelligan, lui, se disait Canadien français, était en fait le premier poète engagé du Québec" (Jacques Ferron, dans les notes inédites que j'ai prises lors d'une entrevue publiée dans Lettres Québécoises, no 6 (avril-mai 1977). p. 34-41.

28. Marius Barbeau, le Rêve de Kamalmouk, Fides, 1948, p. 27. Le docteur Ferron croit que ce roman "est un des plus beaux livres de notre littérature" (Jacques Ferron, Escarmouches, la longue passe, t. II, p. 26). "Beau" a cause de la présence d'une obsession toute ferronienne: I'avertissement que représente, pour les Québécois, l'abandon des territoires amerindiens aux capitalistes blancs.

29. Chez Ferron, les fougères sont le signe d'un milieu sain. Elles rappellent la nature du passé exempte de la civilisation du béton. Dans Escarmouches, la longue passe, ferron nous montre le vieux docteur Agapit Lupien, déprimé par l'asphalte d'East Greenfield et recevant ses vieux patients comme des créatures poétiques fanées par l'age et par le béton. Le docteur Lupien puise sa raison đ'êtte dans l'imaginaire. Il rêve à des fougères qui se déroulent dans un brouillard vert, qui le plongent dans un passé verdoyant plus humain et moins asphalté It. I, p. 291).

30. "Ne vends pas la terre", Recits laurentiens, Frères des Ecoles chrétiennes, 1942, p. 119.

31. Louis Hémon, Monsieur Ripois et la Némésis, Bernard Grasset, 1967, p. 89.

32. Jacques Ferron, préface de Colin-Maillard, p. 10.

33. Pierre Corneille, Don Sanche D'Aragon (préface de Félix Hérnon).

34. Jacques Ferron, préface de Colin-Maillard, p. 11, p. 24.

35. Ibid., p. 45, p. 54.

36. Jacques Ferron, Du fond de mon arrière-cuisine, p. 21.

37. Voir Jacques Cazotte, le Diable amoureux et autres ecrits fantastiques, Flammarion, 1974, p. 150.

38. Lewis Carroll, Alice in Wonderland, The Children's Press, 1970, p. 156.

39. Jacques Ferron, Escarmouches, la longue passe, t. I, p. 18.

40. Nathaniel Hawthorne, The House of Seven Gables, Houghton Miffiin Company, 1932, p. 30.

41. Ibid., p. 395.

42. Nathaniel Hawthorne, The Scarlet Letter, Oxford University Press, 1965, p. 9-10. 